\title{
Wertung des aktuellen Wissensstands zur CIN
}

\section{Von Prof. Dr. Henrik Michaely, MVZ Radiologie Karlsruhe}

Der wissenschaftliche Fachausschuss der deutschen Ärzteschaft hat vor kurzem einen interessanten Artikel veröffentlicht der die Existenz der Kontrastmittel-induzierten Nephropathie (CIN) in Frage stellt ( > akdae.de > Arzneimitteltherapie > AVP, Ausgabe 4 Okt $2017>$ Übersichtsarbeiten). Dieser Artikel folgt zahlreichen Veröffentlichungen der letzten Jahre, die die Existenz der CIN bei der intravenösen Applikation von Kontrastmitteln im CT grundsätzlich in Frage stellen. McDonald et al konnten in einer Metaanalyse mit mehr als 25000 Patienten im Jahr 2013 keinen Unterschied für das relative Risiko eines akuten Nierenversagens (acute kidney injury - AKI - definiert äquivalent zur CIN als Serkumkreatininanstieg jedoch anders benannt, da auch Patienten ohne KM-Gabe als Kontrollgruppe eingeschlossen wurden), der Notwendigkeit einer Dialyse und für einen Todesfall zwischen Patienten, die eine Untersuchung mit oder ohne Kontrastmittel erhalten haben, feststellen [1]. Auch bei Patienten mit einem Serumkreatinin von $>2 \mathrm{mg} / \mathrm{dl}$ konnte in einer weiteren Studie kein Unterschied in der Inzidenz eines AKI nachgewiesen werden [2]. Zwar steigt die Inzidenz eines AKI mit steigendem Serumkreatinin signifikant von $1,2 \%$ bei einer eGFR $>90 \mathrm{ml} / \mathrm{mim} / 1,73 \mathrm{~m}^{2}$ bis auf $14 \%$ bei einer eGFR $<30 \mathrm{ml} / \mathrm{mim} / 1,73 \mathrm{~m}^{2}$ an, allerdings ohne Unterschiede zwischen den Gruppen der Patienten mit bzw. ohne kontrastverstärkte CT-Untersuchung [3]. Zur Annahme, dass es eine CIN möglicherweise gar nicht gibt, passen auch die Ergebnisse der
AMACING-Studie, die keinen Unterschied für das Auftreten einer CIN/eines AKI nach Kontrastmittelgabe mit oder ohne Wässerung von Patienten mit einer GFR von 30 $59 \mathrm{ml} / \mathrm{mim} / 1,73 \mathrm{~m}^{2}$ nachweisen konnten [4]. Dem entgegen stehen Ergebnisse einer retrospektiven Studie von Davenport et al mit mehr als 13000 Patienten, die zumindest bei einer Subgruppe von Patienten mit einer eGFR $<30 \mathrm{ml} / \mathrm{mim} / 1,73 \mathrm{~m}^{2}$ eine signifikante Erhöhung des AKI-Risikos bei der KM-CT Gruppe verglichen zur nativen CT-Gruppe um einen Faktor 2,96 nachweisen konnte [5].

Was bedeuten diesen Studienergebnisse für uns? Zum einen zeigen die Studien, dass eine klare Trennung der Risikogruppen am besten nach eGFR erfolgen sollte, damit eine sichere Abgrenzung der Patienten mit einer eGFR $<30 \mathrm{ml} / \mathrm{mim} / 1,73 \mathrm{~m}^{2}$ erfolgen kann. Zum anderen scheint das Risiko einer CIN - so sie denn wirklich existiert - deutlich kleiner zu sein als bisher angenommen, in den Niedrig-Risikogruppen mit einer eGFR > $30 \mathrm{ml} / \mathrm{mim} / 1,73 \mathrm{~m}^{2}$ möglicherweise sogar null [6]. In der verbleibenden HochRisikogruppe mit einer eGFR $<30 \mathrm{ml} / \mathrm{mim} /$ $1,73 \mathrm{~m}^{2}$ ist die Faktenlage nicht ganz klar. Hier sollte weiterhin die Notwendigkeit einer Kontrastmittelgabe im CT und der mögliche Erkenntnisgewinn gegenüber dem Risiko einer CIN abgewogen werden, selbst wenn bei Patienten mit AKI/CIN und eGFR $<30 \mathrm{ml} / \mathrm{mim} / 1,73 \mathrm{~m}^{2}$ keine Mortalitätsunterschiede nachweisbar waren [2].

\section{Literatur}

[1] McDonald JS, McDonald RJ, Comin J et al. Frequency of acute kidney injury following intravenous contrast medium administration: a systematic review and meta-analysis. Radiology 2013; 267: 119-128 doi: 10.1148/radiol.12121460. Epub 2013 Jan 14. Review

[2] McDonald RJ, McDonald JS, Carter RE et al. Intravenous contrast material exposure is not an independent risk factor for dialysis or mortality. Radiology 2014; 273: 714-725 doi: 10.1148/radiol.14132418. Epub 2014 Sep 9

[3] McDonald JS, McDonald RJ, Carter RE et al. Risk of intravenous contrast material-mediated acute kidney injury: a propensity scorematched study stratified by baseline-estimated glomerular filtration rate. Radiology 2014; 271: $65-73$ doi: 10.1148/radiol.13130775. Epub 2014 Jan 16.

[4] Nijssen EC, Rennenberg RJ, Nelemans PJ et al. Prophylactic hydration to protect renal function from intravascular iodinated contrast material in patients at high risk of contrastinduced nephropathy (AMACING): a prospective, randomised, phase 3 , controlled, open-label, non-inferiority trial. Lancet 2017; 389: 1312 - 1322 doi: $10.1016 /$ S01406736(17)30057-0. Epub 2017 Feb 21

[5] Davenport MS, Khalatbari S, Dillman JR et al. Contrast material-induced nephrotoxicity and intravenous low-osmolality iodinated contrast material. Radiology 2013; 267: 94-105 doi: 10.1148/radiol.12121394. Epub 2013 Jan 29

[6] McDonald RJ, McDonald JS, Newhouse JH et al. Controversies in Contrast Material-induced Acute Kidney Injury: Closing in on the Truth? Radiology 2015; 277: 627-632 doi: 10.1148/ radiol.2015151486. Review. No abstract available 